\title{
La surveillance de l'environnement associée aux essais nucléaires en Polynésie française
}

\author{
R. COULON ${ }^{1}$, \\ C. ARNOULD* ${ }^{*}$ P. BORY ${ }^{\dagger}$, R. DUCOUSSO* ${ }^{*}$ J. SARBACH*
}

(Manuscrit reçu le 27 mars 2009, accepté le 18 avril 2009)

RÉSUMÉ La réalisation du programme d'expérimentations nucléaires en Polynésie française a été accompagnée par la mise en place d'un dispositif de surveillance radiologique de l'environnement, qu'il s'agisse du milieu physique ou biologique, continental ou marin, végétal ou animal, afin de s'assurer de l'absence de risque pour les populations. Ce dispositif a pris toute son importance avec les essais réalisés dans l'atmosphère entre 1966 et 1974. Sa mise en œuvre a nécessité des moyens importants en matériel et en personnel avec l'intervention d'organismes civils et militaires ou parfois mixtes. La surveillance a naturellement été exercée de façon très complète dans les zones voisines des sites de tir, mais aussi sur l'ensemble du territoire polynésien, notamment les atolls les plus peuplés. Elle a porté également, mais de façon plus allégée, sur des zones lointaines, comme les pays andins, en raison de leur situation géographique. Les résultats obtenus par les différents laboratoires impliqués étaient rassemblés et interprétés afin d'assurer l'information des responsables et des autorités, notamment en Polynésie, et à travers eux la population. Ils ont été chaque année transmis au comité scientifique des Nations-Unies pour l'étude des effets des rayonnements ionisants (UNSCEAR). Le dispositif de surveillance est ainsi décrit, en dehors des zones proches des zones de tir, avec la présentation des différents organismes impliqués et celle des différents programmes de surveillance.

ABSTRACT Environmental monitoring associated with nuclear testing in French Polynesia.

The realization of the programme of nuclear experiments in French Polynesia was accompanied by the installation by a radiological monitoring device of the environment, either the physical environment or biological, continental or marine, vegetable or animal, in order to make sure of the absence of risk for the populations. This device took all its importance with the tests carried out in the atmosphere between 1966 and 1974. Its implementation required important means in hardware and manpower with the intervention of civil and military or sometimes mixed organizations. The monitoring was naturally done in a very complete way in the zones close to the sites of tests, but also on the whole of the Polynesian territory, in particular the most populated atolls. It also carried, but in a less extended way, on remote zones, like the Andean countries, because of their geographical location. The results obtained by the various involved laboratories were gathered and interpreted in order to ensure information of the persons in charge and the authorities, in particular in Polynesia and through them the population. They were each year

Société française de radioprotection, BP 72, 92263 Fontenay-aux-Roses Cedex, France.

* Les auteurs ont été impliqués à des titres divers dans la réalisation du programme de surveillance faisant l'objet de cette présentation : R. Coulon, rédacteur de cet article, au titre du Département de protection sanitaire, C. Arnould et J. Sarbach au titre de l'Institut de protection et de sûreté nucléaire, R. Ducousso au titre du Service mixte du contrôle biologique et P. Bory comme expert du CEA. 
transmitted to the scientific committee of the United Nations for the study of the effects of ionizing radiations (UNSCEAR). The surveillance device is thus described, apart from the zones close to the zones of testing, with the presentation of the various implied organizations and the various monitoring programs.

Keywords: Nuclear testing / radiation monitoring / environment

Dans le passé, la France a développé un programme d'expérimentations nucléaires qui a débuté au Sahara par quatre essais de faible puissance dans l'atmosphère (Reggane) en 1960 et 1961 et treize essais souterrains (In Ecker) entre 1961 et 1966. Ensuite, le programme s'est poursuivi en Polynésie française (atolls de Mururoa et Fangataufa) avec 41 essais dans l'atmosphère entre 1966 et 1974 et 137 essais souterrains entre 1975 et 1996, date à laquelle le programme a pris fin définitivement. Tous ces essais ne représentent qu'une faible partie de l'ensemble de ceux qui ont été réalisés dans le monde depuis 1952, essentiellement par les États-Unis et l'ex-URSS.

Les aspects radiologiques associés à la réalisation d'un programme d'expérimentations nucléaires ne peuvent être ignorés, d'autant qu'ils ne concernent pas seulement la nation sur le territoire de laquelle ils sont effectués, mais peuvent aussi concerner d'autres pays lorsqu'il s'agit d'essais réalisés dans l'atmosphère. Concernant le programme français en Polynésie ces aspects sont traités de façon particulièrement complète et bien documentée dans trois rapports de référence qui servent de base à l'information dans ce domaine. Il s'agit d'un rapport de l'Office parlementaire d'évaluation des choix scientifiques et technologiques (OPECST), publié en 2002 (Bataille et Revol, 2002), un rapport du ministère de la Défense publié en 2007 (Ministère de la Défense, 2007) et d'un rapport du Commissariat à l'énergie atomique publié également en 2007 sous le titre «Les atolls de Mururoa et de Fangataufa (Polynésie française). Les expérimentations nucléaires, aspects radiologiques » (Martin, 2007).

Ces rapports traitent bien évidemment des aspects environnementaux. Toutefois étant donné la très grande importance qu'ils peuvent revêtir et en raison de l'ampleur du programme de surveillance mis en place, nécessitant des règles strictes d'organisation et de fonctionnement, impliquant de nombreux acteurs et conçus différemment selon l'objectif à atteindre, il a paru utile de présenter ce programme dans son ensemble et de façon aussi complète que possible.

Cet article porte donc essentiellement sur les essais nucléaires réalisés dans l'atmosphère en Polynésie française entre 1966 et 1974, en excluant la surveillance exercée au niveau des sites de tirs ainsi que la présentation des résultats, ces deux derniers sujets étant traités dans les rapports mentionnés ci-dessus que le lecteur peut consulter. 


\section{Quelques rappels}

\subsection{Les essais nucléaires}

L'explosion d'un engin nucléaire s'accompagne de la production de très nombreux radionucléides. Il s'agit pour l'essentiel de ceux qui sont générés par la réaction de fission (les fragments des produits de fission) auxquels s'ajoutent ceux qui résultent de l'action des neutrons sur les différents matériaux (les produits d'activation). Avec le reste de la matière fissile non consommée, cet ensemble constitue une masse radioactive très importante quelle que soit la puissance de l'explosion.

Dans le cas des expérimentations souterraines, cette masse radioactive reste confinée dans la cavité où a eu lieu l'explosion, sauf, bien entendu, en cas de fuite accidentelle. Par contre, dans le cas des essais aériens, elle est propulsée extrêmement violemment dans l'atmosphère puis s'élève dans la troposphère et dans la stratosphère puisqu'elle peut atteindre une altitude d'une vingtaine de kilomètres. Les phénomènes mécaniques et thermiques qui conditionnent la formation et l'évolution de ces phénomènes, ainsi que la répartition de la radioactivité au sein de l'atmosphère sont décrits par ailleurs (Ministère de la Défense, 2007 ; Martin, 2007).

Le devenir des radionucléides dans l'environnement peut être représenté par la séquence :

$$
\text { injection } \rightarrow \text { dispersion } \rightarrow \text { dépôt. }
$$

Les cheminements suivis diffèrent toutefois selon la taille des particules formées par les divers matériaux qui sont pulvérisés au sein du nuage lors de l'explosion et qui servent de supports aux éléments radioactifs.

La fraction associée aux particules les plus lourdes se disperse sous l'action du vent dans les basses couches de l'atmosphère et se dépose assez rapidement sur des distances de quelques dizaines voire centaines de $\mathrm{km}$ au-delà du site de tir. C'est la retombée locale, ou régionale, sans doute la plus contaminante, qui comporte la plupart des radionucléides en particulier les radionucléides à vie courte comme l'iode-131. Elle se produit dans les heures, voire les jours, qui suivent le tir mais n'affecte que des zones limitées.

Une autre fraction constituée d'aérosols plus fins se retrouve à plus haute altitude et, sous l'action entre autres des courants aériens, suit une trajectoire plus ou moins longue mais qui peut l'amener à faire le tour, voire plusieurs tours, de la Terre dans l'hémisphère où a eu lieu le tir. Tout au long de cette trajectoire, les 
radionucléides redescendent lentement à la surface des zones survolées. D'origine troposphérique, cette retombée se produit durant les jours, voire les mois qui suivent le tir et ne comporte déjà plus que peu ou pas de produits à vie courte.

Enfin, une dernière fraction, constituée des aérosols les plus fins, est injectée dans la stratosphère où elle se redistribue de façon plus large et plus homogène essentiellement dans l'hémisphère où a eu lieu le tir en raison du peu d'échanges entre les deux hémisphères. Elle s'en élimine lentement en repassant dans la troposphère et en se déposant au sol. Le temps de résidence moyen est de quelques mois à quelques années et le dépôt, constitué essentiellement de radionucléides à vie longue, peut couvrir une part importante de l'hémisphère.

Dans tous les cas, les radionucléides qui atteignent la surface du sol se retrouvent dans les différents compartiments de l'environnement, en particulier, par le jeu des transferts, dans les productions végétales et animales qui entrent dans l'alimentation humaine. La question des transferts dans l'environnement a été abondamment traitée dans le cadre similaire des rejets, concertés ou accidentels, des installations nucléaires et celui des études radioécologiques.

\subsection{La surveillance de l'environnement}

La protection de la population vis-à-vis des conséquences potentielles des retombées dues aux essais nucléaires dans l'atmosphère passe d'abord par la recherche d'une minimisation de ces retombées et des risques d'atteinte de la population.

Parmi les principales dispositions, on peut citer :

- le choix des sites de tir, situés au plus loin des zones habitées,

- le choix des caractéristiques et des conditions météorologiques les plus favorables, à savoir celles qui évitent que la trajectoire du nuage passe sur des zones habitées,

- le choix du mode de tir, sachant que lorsqu'un tir est effectué à faible hauteur la boule de feu atteint la surface sous-jacente et entraîne des matériaux pulvérisés qui constituent alors des vecteurs pour le dépôt des radionucléides.

La situation géographique des atolls de Mururoa et de Fangataufa, inhabités à l'origine, va dans ce sens : ils sont situés dans une zone très peu peuplée (environ 2300 et 5000 habitants respectivement dans un rayon de 500 et $1000 \mathrm{~km}$ ), très loin de Tahiti, l'île la plus peuplée et le régime des vents a pour effet d'entraîner le nuage principal vers l'Est, survolant ainsi une zone maritime quasi désertique, le continent sud américain se trouvant à plus de $6000 \mathrm{~km}$ dans cette direction. Par ailleurs, la technique du tir sous ballon a permis d'éviter le léchage de la surface de l'atoll par la boule de feu. 
En dépit des précautions prises au niveau des tirs, une surveillance de l'environnement s'avère indispensable, puisqu'elle permet de s'assurer de l'absence de risque radiologique pour les populations et, au cas où un tel risque serait mis en évidence, de l'évaluer et de prendre à temps les mesures nécessaires pour y parer.

C'est pourquoi les autorités ont décidé de mettre en place un vaste programme de surveillance de l'environnement, qu'il s'agisse du milieu physique et du milieu biologique, continental et marin, proche et lointain. L'organisation et les modalités de ce programme ont été définies en 1962. Le gouvernement a notamment veillé à une répartition des tâches entre les organismes impliqués dans sa mise en œuvre, en particulier les organismes militaires et civils, afin d'éviter toute critique qui pourrait naître d'une situation de monopole par l'un ou l'autre des deux organismes. Il était cependant important que la coordination entre ces organismes soit parfaite, qu'il s'agisse des problèmes de matériel, de financement, de qualité des résultats, de transmission des informations, d'évolution des programmes en fonction du contexte et d'exploitation des résultats.

\section{Les principaux acteurs}

La mise en œuvre du programme a fait appel à des structures existantes, déjà impliquées dans des actions de surveillance radiologique, tant au niveau de la surveillance générale du territoire métropolitain qu'au niveau du programme d'expérimentations nucléaires au Sahara, et donc parfaitement qualifiées. Mais l'expérience acquise au Sahara et la spécificité des problèmes posés en Polynésie (ampleur du programme, éloignement de la métropole, particularités du milieu, notamment l'importance du milieu marin, réactions de la population et du milieu international, ...) ont conduit à créer d'autres structures mieux adaptées à cette situation nouvelle. Ces structures sont présentées ci-dessous.

\subsection{Les précurseurs}

Au CEA existait le département de protection sanitaire (DPS) créé en 1961 et dont les missions comprenaient aussi la surveillance de l'environnement.

Ce département disposait notamment de laboratoires compétents et reconnus, pour les analyses et mesures de radioactivité sur des échantillons variés appartenant au milieu biologique et au milieu physique, à savoir :

- un laboratoire de radiotoxicologie (LRT), basé à Fontenay-aux-Roses sur le site du Centre d'études nucléaires, compétent pour effectuer des mesures fines de radioactivité sur les échantillons biologiques, végétaux et animaux, 
- un laboratoire de mesures de la radioactivité de l'air (LMRA), basé à Orsay sur le site de la faculté des sciences, spécialisé en métrologie des échantillons du milieu physique (poussières atmosphériques, eau de pluie, ...), qui s'est transformé en laboratoire de métrologie relatif à l'environnement (LMRE).

L'organisation et la dénomination du département ont été modifiées au cours du temps : en 1976 il fut notamment rattaché à l'Institut de protection et sûreté nucléaire (IPSN), créé à cette date dans le cadre du CEA. Par commodité, il sera désigné par le sigle DPS tout au long de ce document. Il faut ajouter que le DPS disposait également de compétences et de moyens dans le domaine situé en amont des mesures (échantillonnage, acheminement des prélèvements, traitement et préparation des échantillons) et, bien entendu, dans le domaine de l'exploitation et de l'interprétation sanitaire des résultats.

Le DPS était très impliqué dans les actions liées à la surveillance radiologique au niveau du territoire national, notamment du fait des retombées provenant des expérimentations réalisées par d'autres puissances nucléaires. Les personnels et les matériels étaient donc parfaitement adaptés à ce type d'activité.

Du reste, en 1962, une station de surveillance fut implantée à Tahiti. Il s'agissait du groupe de surveillance radiologique (GSR). Il avait pour mission d'effectuer des prélèvements (poussières atmosphériques, précipitations, produits alimentaires) afin d'évaluer le niveau de contamination du milieu, sorte de « point zéro » avant le début des expérimentations françaises. Le GSR sera ultérieurement intégré au laboratoire de surveillance de la radioactivité (LSR) qui deviendra plus tard le laboratoire d'étude et de surveillance de l'environnement (LESE) présenté plus loin.

À signaler également que le Service central de protection contre les rayonnements ionisants (SCPRI), devenu Office de protection contre les rayonnements ionisants (OPRI) en 1994, puis fusionné en 2002 dans l'Institut de radioprotection et de sûreté nucléaire (IRSN), chargé de la surveillance radiologique au niveau national, a implanté en 1984 une station de surveillance à Tahiti. Rattachée au LESE elle portait classiquement sur la surveillance des poussières atmosphériques, des précipitations, du lait et des eaux océaniques.

\subsection{La direction des centres d'expérimentations nucléaires (DirCEN) et ses services}

Aux unités indiquées ci-dessus furent ajoutées des unités nouvelles pour répondre aux besoins liés à la surveillance radiologique de l'environnement associée aux essais. Il s'agit pour le CEA, d'un laboratoire pour la mesure de la radioactivité 
implanté en Polynésie et, pour ce qui est des Armées, de deux services créés au sein d'une structure nouvelle, la DirCEN : ces deux services furent chargés d'assurer la mission de sécurité radiologique et de contrôle biologique.

\subsection{1. $L a \ll \operatorname{DirCEN} »$}

La DirCEN a été créée par un décret du 30 janvier 1964, signé conjointement par le ministre des armées et le ministre d'Etat chargé de la recherche scientifique et des questions atomiques et spatiales : elle était géographiquement implantée dans la zone Louis Breguet de l'aérodrome de Villacoublay dans la région parisienne. C'est à cette direction, composée de personnels des armées et du CEA, qu'a été confiée la responsabilité de la conception, de la création et de l'exploitation des centres de tirs.

Pour remplir la mission de surveillance et de contrôle qui lui incombait, deux services furent créés au sein de la DirCEN :

- le service mixte de sécurité radiologique (SMSR) par arrêté interministériel du 30 juillet 1964 ,

- le service mixte de contrôle biologique (SMCB) par arrêté interministériel du 8 août 1964.

Ces deux services étaient mixtes, c'est-à-dire qu'ils étaient composés de personnels dépendant du ministère de la défense (militaires et civils) et de personnels dépendant du CEA.

En 1984, un comité scientifique pluridisciplinaire composé de personnalités scientifiques extérieures (IPSN, CNRS, Institut Curie, CHU, Muséum d'histoire naturelle, ...) qui avait initialement été créé pour conseiller le SMCB dans l'élaboration des directives et la conduite des opérations nécessaires à l'accomplissement de sa mission, a été mis en place auprès du directeur de la DirCEN.

En 1994, en raison du contexte politique et du moratoire sur les essais nucléaires, les deux services, SMSR et SMCB, ont fusionné et donné naissance au service mixte de surveillance radiologique et biologique de l'homme et l'environnement (SMSRB).

En 1998, la DirCEN et donc le SMSRB, a été dissoute. Ensuite a été créé au sein de la Délégation générale pour l'armement, un département de suivi des centres d'expérimentations nucléaires (DSCEN). 


\subsubsection{Le SMSR}

Sa mission

Le SMSR jouait sur les sites le rôle d'un service de protection contre les rayonnements tel qu'il en existe dans tout centre nucléaire. À ce titre, il était chargé de veiller au respect des règles de protection lors des tirs et d'assurer la surveillance et le contrôle de la contamination du milieu physique (poussières atmosphériques, précipitations, dépôts, sols, eaux océaniques) sur et au voisinage des sites d'expérimentation mais aussi sur l'ensemble du territoire de la Polynésie. Il avait également la responsabilité du contrôle dosimétrique des personnels civils et militaires et intervenait lors des opérations de décontamination.

\section{Son organisation}

La direction du service était basée à Montlhéry au sud de Paris avec une antenne importante à Tahiti (Mahina). Pendant les essais aériens, il disposait aussi d'une antenne à Mururoa, avec un regroupement des moyens sur le bateau de soutien logistique (BSL) Rance, et d'une autre sur l'atoll Hao. Par la suite, après le passage aux essais souterrains, l'essentiel des moyens ont été installés à Mururoa.

Unité dépendant de la DirCEN, le SMSR était composé d'agents militaires et civils, ces derniers relevant de la direction des essais de la direction des applications militaires (DAM) du CEA. Il employait également un personnel polynésien venant essentiellement de Tahiti. Le chef du service et son adjoint étaient alternativement un militaire et un civil.

\section{Ses moyens}

Le SMSR disposait de tous les moyens nécessaires à l'exercice de sa mission, notamment en matière de contrôle et de surveillance. Dans ce domaine, il s'appuyait sur deux laboratoires de mesures très performants, l'un à Montlhéry, l'autre à Tahiti, et pendant les expérimentations aériennes, d'un ensemble de moyens sur le BSL «Rance ». Par la suite, le laboratoire de Tahiti a été implanté à Mururoa. Ces laboratoires effectuaient des mesures permettant d'évaluer une éventuelle contamination d'échantillons prélevés sur milieu physique provenant des sites et de l'ensemble de la Polynésie.

Par ailleurs, pendant la période des expérimentations dans l'atmosphère, des moyens permettant des mesures extemporanées et des prélèvements sur le milieu physique étaient implantés sur l'ensemble du territoire, sur des atolls habités et inhabités : il s'agissait des postes de contrôle radiologique (PCR) et des postes de surveillance radiologique (PSR). Les premiers étaient exploités par des techniciens 
de radioprotection, les seconds pouvaient l'être par des personnels non spécialisés (gendarmerie par exemple). Des moyens de ce type étaient également embarqués sur les différents bâtiments utilisés et sur les avions chargés de mission de détection. Enfin un réseau de télémesures sur atolls inhabités et de mesures par bouées avait été mis en place en zone de tir et en zone proche.

Il est évident que ces moyens, qui constituaient un dispositif impressionnant nécessité par les campagnes d'essais atmosphériques et qui fonctionnaient de façon permanente pour certains alors que d'autres étaient activés pendant la période des tirs, ont été peu à peu réduits après le passage aux tirs souterrains.

L'implantation de ces différents moyens sont présentés figure 1.

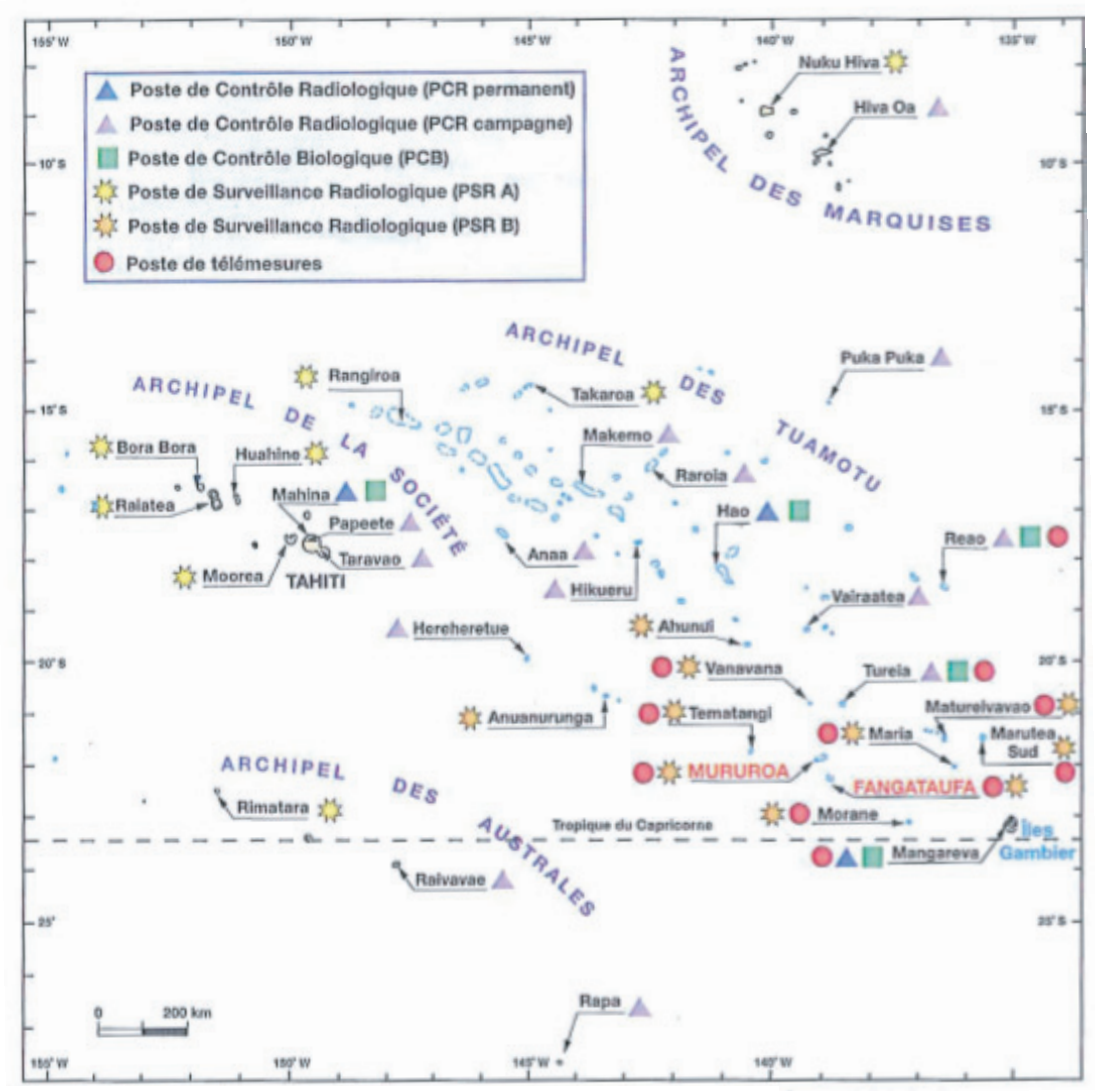

Figure 1 - Localisation des postes de contrôle et de surveillance radiologique et biologique en Polynésie française, lors de la période des essais atmosphériques (Martin, 2007).

Location of checkpoints and radiological and biological monitoring in French Polynesia during the period of atmospheric testing (Martin, 2007). 
R. COULON et al.

\subsubsection{Le SMCB}

\section{Sa mission}

L'arrêté de création, du SMCB le charge :

- de promouvoir et coordonner les recherches, études et mesures de contrôle nécessaires à la surveillance radiologique des zones intéressées par les expérimentations, en faisant appel aux différents organismes scientifiques, civils et militaires, compétents pour exécuter les missions envisagées,

- de proposer toute mesure nécessaire à la sauvegarde de la flore, de la faune et des denrées comestibles.

En résumé, le SMCB avait pour mission d'assurer la surveillance radiobiologique des êtres vivants (à l'exclusion de l'homme, apanage des services médicaux), des denrées alimentaires et des eaux sur les sites d'expérimentations, mais aussi dans l'ensemble de la Polynésie française.

\section{Son organisation}

De 1965 à 1994, l'organisation générale du SMCB a reposé sur deux échelons : l'un en métropole, l'autre en Polynésie française. L'échelon métropolitain était implanté d'abord à Villacoublay auprès de la DirCEN, puis dans le Centre d'études de Montlhéry, sur la commune d'Ollainville près d'Arpajon dans l'Essonne. Il était le siège de la direction générale du service qui comportait un bureau scientifique et un bureau administratif. La direction générale définissait et dirigeait les programmes de prélèvements, ainsi que les études scientifiques indispensables à l'accomplissement du contrôle. Elle exploitait les résultats d'analyse et élaborait les documents de synthèse. Elle fournissait à l'échelon polynésien les moyens correspondants aux missions qu'elle lui confiait. Par ailleurs, l'échelon métropolitain, en la personne du chef du SMCB ou du médecin officier supérieur adjoint, était « conseiller santé » de l'officier général commandant la DirCEN.

L'échelon polynésien comportait une direction locale située à Tahiti-Mahina, qui pilotait directement trois équipes chargées des prélèvements, l'une à Mururoa, une autre à Tahiti-Mahina, la troisième à bord d'un bâtiment de contrôle biologique.

\section{Ses moyens}

Les personnels du SMCB relevaient du ministère de la défense et du CEA. La participation du CEA importante à sa création, s'est réduite au fil du temps et ne comportait plus en 1986 qu'un ingénieur basé à Montlhéry. La direction de l'échelon polynésien, jusqu'alors assurée par un médecin de l'IPSN, a été ensuite confiée à un médecin du service de santé des armées (SSA). 
La majorité des agents de ce service relevaient du SSA (médecins, pharmaciens, vétérinaires, officier chargé des tâches administratives du service de santé des armées), mais on comptait aussi parmi eux des électroniciens, des techniciens spécialistes de la métrologie... Les Armées avaient également fait appel à des spécialistes de biologie et d'océanographie recrutés comme personnel civil de la défense (PCD).

En Polynésie, un personnel local avait été embauché pour assurer les missions de prélèvements et certaines tâches administratives ou d'entretien des matériels et des installations.

À Tahiti et à Mururoa, le SMCB possédait les matériels (embarcations) et les équipes permettant d'effectuer les prélèvements terrestres et marins. Pour effectuer les prélèvements océaniques et les prélèvements terrestres dans les archipels lointains, il disposait d'un bâtiment adapté à cette mission : ce bâtiment fut d'abord le bâtiment de recherche océanographique (BRO), la «Coquille» armé et géré par la Marine nationale et affrété par le SMCB. La gérance « maritime » donnait lieu à des conflits de subordination notamment lors du temps consacré à l'entraînement militaire de l'équipage. Aussi, en 1973, la Coquille fut elle remplacée par le «Marara », construit spécialement pour les besoins du service. Il était armé par la DirCEN, géré par la compagnie générale maritime (CGM) et affrété par le SMCB ou le SMSR après le retrait de la Rance. Son équipage était polynésien hormis le commandant, le second, le radio et le mécanicien. Il était gréé pour la pêche à la longue ligne, à la traîne et au chalut.

Le gouvernement, afin que le contrôle biologique ne soit pas le monopole d'un seul organisme, n'a pas doté le SMCB de laboratoires d'analyse. Les échantillons prélevés étaient confiés aux laboratoires de son choix. Depuis l'origine, le principal des analyses a été effectué par le laboratoire implanté par le CEA en Polynésie française.

Le SMCB confiait également partie de ses échantillons à d'autres laboratoires. Pendant longtemps, ce fut la division de radiobiochimie du centre de recherches du service de santé des armées (CRSSA). Ensuite ce fut le SMSR, le service de protection radiologique des armées (SPRA), et le laboratoire de radiobiologie du Pacifique.

Depuis 1965, date de l'implantation de son échelon polynésien, le SMCB a dû adapter les modalités du contrôle radiobiologique aux conditions de réalisation des essais, tout en respectant les termes de son arrêté de création. Les procédures ont été mises sur pied en fonction des essais souterrains réalisés d'abord à partir de la couronne émergée des atolls, puis dans la zone centrale lagunaire. L'adaptation la 
plus drastique a correspondu au passage du contrôle des essais atmosphériques à celui des essais souterrains en 1975. Il s'est agi en fait d'une véritable restructuration débouchant sur une division par deux des effectifs, en liaison avec une division par deux du nombre d'échantillons prélevés.

\subsection{Le laboratoire de surveillance radiologique en Polynésie}

Création, mission, évolution

C'est en 1962, après une mission d'exploration des Tuamotu destinée à choisir un site propice à des expérimentations nucléaires que le chef du DPS au CEA, décide d'installer à Tahiti une station locale de prélèvements du milieu biologique et du milieu physique pour la surveillance de la contamination de l'environnement : ainsi est né le groupe de surveillance radiologique (GSR).

Cette unité, reliée aux laboratoires métropolitains du DPS se verra confier une double mission :

- effectuer une surveillance de la contamination due aux essais étrangers (en particulier américains),

- fournir «un point zéro» avant que ne débutent les campagnes de tirs atmosphériques sur les atolls de Mururoa et Fangataufa.

Le rôle du GSR consistait à gérer la collecte d'échantillons biologiques qui, après calcination, étaient envoyés au laboratoire de radiotoxicologie du DPS pour analyses et mesures et les prélèvements de poussières atmosphériques au laboratoire de métrologie relative à l'environnement également du DPS.

Lorsque furent créés en 1964 le SMSR et le SMCB, le chef du DPS concrétisa, non sans quelques difficultés, l'idée de créer un laboratoire unique, indépendant et ouvert à tous, basé en Polynésie et chargé d'effectuer toutes les mesures sur les échantillons biologiques prélevés par le SMCB, coupant court ainsi à toutes les accusations de dépendance de la surveillance vis-à-vis de l'exécution des expérimentations. Ainsi naquit le Laboratoire de surveillance radiologique (LSR), rattaché au DPS, auquel fut également confiée la gestion de la station locale initiale (ex-GSR).

Dans la pratique, il y eut quelques entorses à la règle voulant que toutes les mesures effectuées sur les échantillons biologiques du SMCB soient réalisées par ce laboratoire. D'une part, parce que le SMCB disposait sur le navire de contrôle biologique, d'un petit laboratoire de mesures $\gamma$ et d'autre part en raison de la saturation du laboratoire polynésien due au grand nombre d'échantillons prélevés et enfin parce que les laboratoires métropolitains, et tout particulièrement au DPS, étaient mieux armés pour l'exécution de mesures fines. 
Il faut ajouter qu'à partir de 1974, l'action du LSR fut également orientée vers une assistance au territoire dans le domaine non nucléaire (analyses physiques et chimiques, contrôles sanitaires, problèmes d'aménagement de l'environnement, applications agricoles, hydrologie, ...) et devint en 1979 le LESE. À partir de 1985, compte tenu de la réduction des programmes de surveillance, le LESE a pris en charge les analyses et mesures effectuées sur les échantillons biologiques du réseau propre au DPS jusqu'alors adressés au laboratoire du DPS à Fontenay-auxRoses.

\section{Les moyens}

Les moyens dont disposait le GSR, puis le LSR et enfin le LESE ont considérablement évolué au cours du temps aussi bien en ce qui concerne le personnel que le matériel.

Le personnel était composé d'agents CEA, ingénieurs et techniciens et de personnel local utilisé pour des tâches diverses (secrétariat, préparation des échantillons, entretien, ...). Des compléments étaient obtenus de façon très ponctuelle par des scientifiques du contingent, des volontaires d'assistance technique (VAT) (service militaire) et des contrats locaux.

Les installations comportaient le matériel classique d'un laboratoire d'analyses et mesures. S'y ajoutait le matériel nécessaire pour les prélèvements marins (bateaux, ...).

Le financement était assuré par le budget du CEA (IPSN par la suite) et par des crédits délégués par la DirCEN et provisionnés par la DAM pour couvrir les analyses et mesures effectuées pour le compte du SMCB.

Après l'arrêt définitif des essais nucléaires en Polynésie et, par voie de conséquence, la dissolution du SMSRB, il fut décidé de poursuivre une surveillance radiologique du territoire et donc de conserver le LESE qui devint en 1999 le laboratoire d'étude et de suivi de l'environnement, rattaché au service d'étude et de surveillance de la radioactivité appartenant à la direction de l'environnement et de l'intervention de l'IRSN (ex-IPSN). Le programme de surveillance assigné à la station et les modalités de sa mise en œuvre n'ont pas été modifiés sensiblement en dépit d'une réduction du personnel métropolitain.

\section{Organisation de la surveillance}

Comme on l'a vu au paragraphe 1.1, on considère que les conséquences d'essais nucléaires dans l'atmosphère peuvent s'observer aux niveaux local, régional et mondial, la répartition de la contamination dépendant très fortement de la 
puissance des tirs, du mode de tir et des conditions météorologiques au moment du tir et dans la période qui suit. Les retombées tendant à être de moins en moins importantes au fur et à mesure que l'on s'éloigne de la zone de tir, il est logique de proportionner l'importance du programme de surveillance à cet état de fait.

Dans le cas des tirs polynésiens, la zone proche soumise à une surveillance locale est constituée par les deux atolls de Mururoa et de Fangataufa et la zone marine avoisinante. Cette surveillance, exercée de façon très complète par le SMSR et le SMCB ne sera pas traitée ici. Il convient cependant de rappeler que les premiers tirs, entre 1966 et 1968, ont été effectués sur barge, et que ce mode de tir était relativement contaminant pour la zone locale. À partir de 1968 ils ont été effectués sous ballon captif, technique plus délicate mais moins contaminante dans la mesure où elle évite le contact entre la boule de feu et la surface du lagon.

La surveillance dite régionale concernait l'ensemble de la Polynésie française, même si certains archipels étaient très éloignés des sites de tir et des trajectoires prévisibles suivies par les masses d'air contaminées. Il a toutefois été observé qu'en dehors de la trajectoire principale du nuage, globalement sur un axe ouestest, se formaient des nuages dérivés, extraits du nuage principal. C'est donc sur l'ensemble de la Polynésie, et particulièrement pour l'île la plus habitée, l'île de Tahiti, que la surveillance a été effectuée de la façon la plus rigoureuse.

La surveillance à l'échelle mondiale ne pouvait être exercée que d'une façon plus simplifiée. Elle ne faisait que compléter la surveillance effectuée par de nombreux pays sur leur propre territoire dont les résultats, exploités par le comité scientifique des nations unies pour l'étude des effets des rayonnements ionisants (l'UNSCEAR), fournissaient un schéma global des retombées imputables à l'ensemble des essais effectués. Il a été jugé nécessaire de compléter ces observations par un réseau général de stations permettant éventuellement d'observer et de suivre la trajectoire correspondant au nuage principal et, le cas échéant, d'être en mesure de détecter une éventuelle situation anormale. Dans ce réseau, une surveillance particulière a toutefois été mise en place dans cinq pays andins bordant la côte ouest du continent sud-américain, car c'était la zone susceptible d'être la première touchée par le nuage principal compte tenu de la direction générale de sa trajectoire.

\subsection{La surveillance de la Polynésie française}

C'est bien entendu la région où la surveillance radiologique se devait d'être la plus complète et la plus affinée avec un triple objectif :

- détecter le plus rapidement possible toute éventuelle augmentation de la contamination : c'est en particulier le rôle de la surveillance atmosphérique, 
- suivre l'évolution à moyen et long terme de la radioactivité présente dans l'environnement, ce qui nécessite la continuité du contrôle dans des conditions normalisées,

- évaluer l'exposition subie par la population du fait des différentes voies d'atteinte et spécialement la voie alimentaire, en cherchant à quantifier la part revenant aux essais français.

L'ensemble des structures mentionnées au chapitre précédent a pris part au programme de surveillance : il était donc nécessaire que règne entre elles, ainsi qu'entre les échelons polynésiens et métropolitains, une parfaite coordination. Une autre nécessité était de surveiller tous les éléments du milieu physique et biologique.

\subsubsection{Surveillance du milieu physique}

\subsubsection{Le milieu atmosphérique}

Très importante au niveau des sites de tirs et des zones proches ou relativement proches pour détecter toute situation qui, principalement lors des tirs aériens, pourrait donner lieu à des conséquences radiologiques, la surveillance atmosphérique était également indispensable dans les zones habitées plus lointaines pour évaluer le niveau de radioactivité et en suivre l'évolution.

En ce domaine, l'action du SMSR était essentielle à travers le réseau de stations présenté au paragraphe 2.2.2. À côté des moyens qui permettaient d'effectuer des mesures en continu avec, si nécessaire, une télétransmission des résultats, d'autres permettaient d'effectuer des prélèvements de poussières atmosphériques qui étaient envoyés au laboratoire (Mahina puis Mururoa) pour des mesures globales ou des mesures fines de radionucléides identifiés.

Les appareils utilisés étaient des appareils classiques de prélèvement sur filtre d'aérosols atmosphériques avec des débits variables allant de 1 à $1000 \mathrm{~m}^{3} / \mathrm{h}$. La fréquence des prélèvements et des mesures était évidemment variable selon la localisation de la station et l'objectif visé.

Pour un suivi constant et à long terme visant la protection des populations, chaque archipel possédait au moins une station de surveillance. Il s'agissait de : Hivaoa, Pukapuka, Rapa, Heretue, Anaa, Raevavae, Raiatea et Bora Bora.

Le DPS possédait également une station de surveillance atmosphérique, mise en place en 1962 (le GSR) et gérée par le LESE. Elle disposait classiquement d'un appareil de prélèvement de poussières atmosphériques. Implantée au départ à proximité de l'aéroport (Tahiti-FAA), elle fut rapatriée plus tard au laboratoire à 
Mahina. Les filtres étaient relevés tous les cinq jours et envoyés pour mesure au laboratoire du DPS à Orsay.

Enfin, comme cela a été signalé, le SCPRI disposait d'une station équipée d'un appareillage de même type. La gestion en était assurée par le personnel du LESE et les mesures effectuées par le laboratoire du SCPRI au Vésinet.

\subsubsection{Les dépôts et les sols}

L'évaluation du dépôt au sol des radionucléides présents dans l'atmosphère passe par la collecte des retombées, celles-ci s'effectuant par voie humide (précipitations) et par voie sèche. On utilise pour cela un « collecteur de retombées des aérosols et des précipitations atmosphériques liquides » (CRAPAL) dont la surface collectrice recueille aussi bien le dépôt sec que les pluies, ces dernières étant passées dans des résines échangeuses d'ions pour la fixation des radionucléides présents.

Pratiquement toutes les stations équipées pour la surveillance atmosphérique possédaient ces appareils. Les prélèvements étaient mensuels ou bimensuels et les mesures effectuées dans les laboratoires (Montlhéry, Orsay, Le Vésinet).

Bien que ces mesures permettaient, indirectement, de connaître la radioactivité du sol, celle-ci faisait également l'objet d'une surveillance par le SMSR, essentiellement sur les sites de tir et les zones proches.

Des prélèvements et mesures, en principe annuels, ont cependant été effectués à Tahiti et d'autres îles avec le souci de différencier la part de la radioactivité (essentiellement le ${ }^{137} \mathrm{Cs}$ et le plutonium) présente en surface $(2 \mathrm{~cm})$ et le profil de sa distribution en profondeur (4 couches de $10 \mathrm{~cm})$.

\subsubsection{Les eaux océaniques}

C'est toujours le SMSR qui avait la charge de la surveillance des eaux océaniques avec un maillage plus étroit en zone proche du site de tir. Néanmoins, des prélèvements étaient effectués au niveau de l'archipel de la Société, des Australes, des Marquises et des Gambiers. Pour les eaux de surface, ils étaient réalisés par pompage à quelques mètres de profondeur et par bouteilles hydrographiques pour les plus grandes profondeurs. Comme pour les sols les très faibles concentrations des radionucléides recherchés $\left({ }^{137} \mathrm{Cs}, \mathrm{Pu}\right)$ nécessitaient des volumes d'eau importants. La périodicité était annuelle.

\subsubsection{Surveillance du milieu biologique}

Qu'il soit terrestre ou marin, la surveillance du milieu biologique revêt une grande importance car c'est à partir de la contamination des produits, végétaux et 
animaux, et de la part que ceux-ci prennent dans le régime alimentaire que peut être évaluée l'exposition résultante pour la population. Les difficultés pour réaliser cette surveillance dans le cas de la Polynésie étaient de plusieurs ordres : diversité des productions et différences selon les îles, variabilité des périodes de production et des quantités produites, large répartition géographique et parfois rareté des moyens de communication.

Les produits collectés étaient essentiellement des produits servant à l'alimentation d'origine locale. Certains étaient parfois des «indicateurs biologiques » c'est-à-dire des végétaux qui, en raison de leur morphologie, captaient mieux les retombées. Des contrôles étaient aussi parfois effectués sur des produits importés dont la contamination était en général plus élevée que celle des productions locales, surtout s'ils provenaient de l'hémisphère nord.

Dans la mesure où des choix étaient possibles, et où, compte tenu des faibles productions, il n'était pas préjudiciable à l'alimentation locale, cet échantillonnage des productions cherchait à représenter les catégories suivantes :

- le lait, encore que la production de lait était très limitée, réservée pratiquement à Tahiti ;

- les légumes

- légumes feuilles (salades, choux, ...),

- légumes racine (taro, patates douces, manioc, ...),

- légumes fruits et fruits (banane, mangue, coco, tomates, ...) ;

- les viandes (porc, poulet, ...) ;

- les produits de la mer

- poisson (chirurgien, perroquet, congre, ...),

- mollusques (bénitier, ...),

- crustacés (crevette, langouste).

La surveillance exercée sur ces produits passait par deux réseaux distincts et complémentaires : le réseau du SMCB et le réseau du LESE/DPS. Le SMCB, outre la surveillance qu'il exerçait en zone proche des sites de tir, effectuait également des campagnes de prélèvements en zone lointaine, c'est-à-dire sur l'ensemble du Territoire grâce à son navire océanographique (la Coquille puis le Marara). L'équipement de ce navire permettait d'effectuer une grande diversité de prélèvements dans le domaine marin : poissons, en particulier poissons de haute mer et poissons vivant en grande profondeur (grâce à la pêche à la « longue ligne »), plancton, crustacés, ... Au cours de ces campagnes des prélèvements étaient effectués dans le milieu terrestre au niveau des différents archipels. Ces prélèvements, végétaux et animaux, entraient dans la liste présentée ci-dessus, dans la mesure où ils correspondaient à des productions existantes dans les îles 
visitées au moment de la campagne. La fréquence des prélèvements était donc fonction de la fréquence des passages.

Les échantillons récoltés pouvaient faire immédiatement l'objet d'une mesure de spectrométrie $\gamma$, exception à la règle qui voulait que les mesures soient confiées à un laboratoire ne dépendant pas du SMCB : le Marara disposait en effet de l'équipement lui permettant, pendant les essais aériens, de déterminer les zones de retombées.

Ramenés à Tahiti et remis au LESE, les échantillons étaient ensuite conditionnés, préparés (dessiccation, minéralisation ...) et mesurés. Les protocoles de mesure seront présentés brièvement plus loin : ce sont les protocoles classiques utilisés dans ce domaine par le DPS. Les résultats étaient ensuite transmis au SMCB qui en assurait la gestion et l'exploitation.

Avec l'arrêt des essais aériens, le programme de surveillance a été peu à peu allégé mais maintenu pour permettre un suivi global. Il a été stoppé en 1998 avec la dissolution du SMSRB.

Le LESE/DPS, qui effectuait les mesures sur les prélèvements SMCB avait son propre réseau de surveillance. La nature des produits faisant l'objet de la surveillance était semblable à celle des prélèvements du SMCB.

Ils étaient prélevés en six stations, réparties sur l'ensemble du Territoire, à savoir :

O archipel de la Société : Tahiti et Maupiti,

○ archipel des Marquises : Hiva Oa,

○ archipel des Tuamotu : Rangiroa,

$\circ$ archipel des Australes : Tubuai,

o archipel des Gambiers : Mangareva.

Les prélèvements étaient effectués par un personnel local, sauf pour Tahiti où ils étaient effectués directement par le personnel du laboratoire, notamment sur le marché. À Tahiti également, le lait produit sur le plateau de Taravao faisait l'objet d'un suivi régulier bien que consommé en quantité négligeable sous forme de lait cru par la population locale.

Théoriquement mensuelle, la fréquence des prélèvements concernant les îles n'a pas toujours été très régulière. Elle dépendait des disponibilités locales ainsi que des possibilités d'envoi vers le laboratoire de Mahina, leur transport étant assuré par avion (Air Polynésie) dans des conditions souvent difficiles en raison de la mauvaise qualité des emballages et de la négligence du transporteur (colis refusés, parfois oubliés, ...). 
Ils étaient regroupés au niveau du laboratoire, pesés, séchés, minéralisés et envoyés pour analyses et mesures au laboratoire de radiotoxicologie du DPS à Fontenay-aux-Roses. Cette situation a perduré jusqu'en 1985, date à laquelle il fut décidé que le LESE effectuerait toutes les analyses et mesures à l'exception bien sûr des filtres atmosphériques.

Les mesures effectuées, qui étaient aussi celles effectuées par le LESE sur les échantillons SMCB, comportaient d'abord une spectrométrie $\gamma$ permettant de connaître l'activité due au potassium-40 et celle due aux radionucléides artificiels, notamment l'iode-131 et le césium-137. La mesure de l'activité $\beta$ des cendres, déduction faite de celle due au potassium-40 donnait une première indication du niveau de contamination. Elle était suivie d'une mesure de l'activité $\beta$ du précipité d'oxalate qui entraîne les alcalino-terreux et notamment les isotopes du strontium (90 et 89) exprimée en «strontium équivalent ». Si cette activité le justifiait la mesure du strontium-90 était effectuée par remise en solution du précipité d'oxalate et extraction de l'yttrium-90 lorsque l'équilibre entre le strontium-90 et l'yttrium-90 était rétabli.

Tous les résultats étaient ensuite rassemblés au DPS pour leur exploitation. Il faut souligner que la surveillance s'est adaptée au cours du temps au régime alimentaire des polynésiens et à son évolution, grâce à une enquête alimentaire très fouillée, réalisée de 1980 à 1982 par le SMCB, dont l'utilité a été au-delà de la seule surveillance radiologique. Il faut souligner que la surveillance s'est adaptée au cours du temps au régime alimentaire des polynésiens et à son évolution.

\subsection{Le réseau mondial de surveillance}

\subsubsection{Le réseau général}

Dès 1965 , le gouvernement décida de mettre en place un réseau de surveillance à l'échelle mondiale, réseau composé de stations dont le choix devait être dicté par différents critères: localisation susceptible de permettre de suivre la trace principale du nuage compte tenu de la trajectoire probable de celui-ci, absence de surveillance radiologique existante, risque ne pouvant être écarté de rencontrer des problèmes d'ordre radiologique (par exemple présence d'iode-131 dans le lait) ou encore justification par des raisons d'ordre politique. Bien entendu, compte tenu de la taille de l'espace à couvrir, il n'était pas possible de disposer d'un réseau suffisamment dense pour fournir un véritable état radiologique, mais plutôt d'un réseau de suivi et d'alarme avec une évidente connotation politique.

Dans la mesure du possible, il était souhaitable d'installer les stations là où existait une présence française car elles pouvaient être tout à fait « officielles » et les résultats pouvaient figurer dans les rapports ouverts : c'est bien entendu le cas 
de Cayenne, de Nouméa, de La Réunion, mais aussi de bases militaires comme Dakar, Diego Suarez, Djibouti, Mers el Kebir.

Pour d'autres stations, il a fallu faire appel au réseau des ambassades et consulats et donc d'avoir l'appui du ministère des affaires étrangères : ce réseau « officieux » classé, comportait une vingtaine de stations.

\subsubsection{Les acteurs}

Outre le rôle du ministère des affaires étrangères, le principe de fonctionnement a été défini dans une décision du Comité interministériel des sites lointains en date du 25 février 1965 qui précisait que le SMSR «a la charge d'effectuer les prélèvements et les mesures physiques et que l'exploitation des renseignements ainsi recueillis et les mesures biologiques sont confiées au département de protection sanitaire du CEA ». On retrouve ainsi le principe général de la répartition des responsabilités entre les Armées et le CEA avec, pour le CEA, la synthèse et l'exploitation des résultats.

\subsubsection{La mise en place}

Le choix des stations fut effectué par le SMSR et le DPS, chacun cherchant à répondre du mieux possible à son objectif propre : la liste initiale fut remaniée au cours du temps pour tenir compte de l'évolution du contexte, des difficultés rencontrées et des enseignements obtenus en cours de fonctionnement et ceci lors de réunions tenues entre les deux parties. Un questionnaire relatif aux possibilités techniques d'implantation et à la désignation d'un responsable local «stable, discret et sérieux » appartenant au personnel de l'ambassade ou du consulat (pour les stations « affaires étrangères) fut envoyé à toutes les stations.

\subsubsection{Le fonctionnement}

Pour assurer la surveillance du milieu physique, les stations disposaient d'appareils de prélèvement de poussières atmosphériques et de recueils de précipitations. La prise en charge, y compris financière, de la fourniture du matériel, son exploitation et son entretien revenait au SMSR. Les échantillons lui étaient adressés pour mesure. Il était en plus admis que certains échantillons mesurés au SMSR pourraient être transmis au DPS/LMRE pour des mesures complémentaires. Des inter-comparaisons furent effectuées entre SMSR et DPS/LMRE.

Pour ce qui est de la surveillance biologique, sous la responsabilité du DPS, il était demandé la fourniture mensuelle d'échantillons de lait, d'un légume feuilles (salades) et de poissons, ceci bien évidemment lorsqu'il existait une production 
locale. Pour l'envoi de ces échantillons de denrées périssables il était prévu des bidons en matière plastique (2 litres), des sacs également en matière plastique et un conservateur (formol). Des consignes étaient données pour l'ajout de formol et la fermeture hermétique des emballages. Les envois étaient assurés par le canal de la valise diplomatique et il fallait éviter le risque de fuites intempestives (il en survint quand même!) risquant de souiller le courrier diplomatique... Le financement du système était assuré également par le SMSR. Les échantillons biologiques étaient adressés au DPS/LRT qui effectuait les mesures

Enfin, selon le principe de base voulu par les autorités gouvernementales, c'est le DPS qui devait rassembler les résultats des mesures et assurer leur exploitation. Fut donc envisagée une transmission rapide et directe de résultats provisoires permettant une interprétation de première urgence et une orientation, si nécessaire, vers des mesures complémentaires. Par la suite, une transmission mensuelle de résultats confirmés et mis en forme fut effectuée.

Quelques difficultés sont apparues dans la mise en œuvre de cette procédure de transmission des résultats par le SMSR, ce dernier souhaitant ne pas précipiter les choses et avoir systématiquement la couverture DirCEN.

Un autre problème fut celui des circonstances dans lesquelles le DPS serait amené à adresser un message d'alerte (appelé «message supra-norme ») aux autorités gouvernementales en cas de nécessité. Selon la gravité de la situation il s'agissait d'un «message d'alerte » ou d'un «message d'alarme »: des niveaux d'alerte et d'alarme ont été proposés sur la base de l'exposition de la thyroïde (1 à $5 \mathrm{rad}$ ). La procédure restait délicate car, en raison des délais incompressibles, les messages ne pouvaient être diffusés dans un délai inférieur à sept jours, d'où la nécessité, en cas de besoin, de circuits directs rapides.

Ce réseau, activé dès le début de chaque campagne et mis en sommeil à la fin a été mis en place dès la campagne de 1966 et a fonctionné parfois bien au-delà de la période des essais nucléaires.

\subsubsection{Le cas des pays andins}

Les pays andins sur la côte ouest du continent sud-américain étaient, parmi les pays étrangers ceux qui risquaient d'être le plus affectés par les conséquences des expérimentations du Pacifique. En effet, compte tenu de la direction générale ouest-est suivie par la trajectoire principale des masses d'air contaminées, cellesci avaient toutes les chances de venir buter sur la barrière andine. La rencontre avec cette barrière, pouvait donc stopper la majeure partie de ces masses d'air avec pour conséquences des retombées d'autant plus importantes que le régime des 
précipitations y est généralement soutenu. Les pays concernés étaient la Colombie, l’Équateur, le Pérou, le Chili ainsi que la Bolivie.

Ces pays ne disposaient que peu, voire pas de structure, de compétence et d'équipement permettant de mettre en œuvre des programmes de surveillance convenables. Ceci étant, les responsables, alertés par les scientifiques, et au-delà la population, manifestaient une certaine inquiétude et rejoignaient le concert de protestations internationales hostiles aux expérimentations. Il fut donc décidé de leur apporter une aide substantielle, laquelle s'est traduite par :

- la mise à disposition des matériels nécessaires à la réalisation d'un programme de surveillance (prélèvements et mesures),

- l'envoi d'experts qualifiés pour la mise en œuvre du programme et l'interprétation des résultats,

- la formation en France de scientifiques de ces pays.

Le programme d'assistance aux pays andins a débuté en 1969 et il a pris fin en 1976, soit deux ans après l'arrêt des essais aériens. Certains pays ont cependant continué la surveillance de façon autonome.

\subsubsection{Les matériels}

Les matériels nécessaires à la réalisation de la surveillance radiologique ont été fournis à chacun des pays concernés et mis en place au sein d'institutions à vocation nucléaire, à savoir :

- la Commission colombienne de l'énergie atomique à Bogota,

- l'École polytechnique à Quito,

- l'Institut péruvien de l'énergie nucléaire à Lima,

- la Commission chilienne de l'énergie nucléaire à Santiago,

- la Commission bolivienne de l'énergie nucléaire à La Paz.

Il s'agissait, pour l'essentiel, d'appareils de prélèvements de poussières atmosphériques (à fort ou moyen débit), de pluviomètres, d'étuves, de fours et enfin de chaînes pour les mesures $\beta$ et de spectromètres $\gamma$. Un technicien électronicien itinérant du SMSR assurait l'entretien du matériel et l'ensemble de l'opération était financé par le SMSR, le matériel devenant propriété des institutions concernées.

\subsubsection{Les experts}

Des experts français étaient mis à disposition, pendant la durée des campagnes, des organismes mentionnés. Les uns étaient des experts physiciens, il y en avait un dans chaque pays, les autres des experts hygiénistes, il y en avait un pour deux ou trois pays. C'étaient des agents du DPS ou des agents mis à disposition du DPS par 
d'autres unités du CEA. Le DPS se chargeait de leur recrutement, de la définition et du cadrage de leur mission et de l'orientation des programmes de surveillance. Il leur portait assistance en cas de difficultés techniques.

Le ministère des affaires étrangères, via les ambassadeurs des cinq pays assuraient la liaison avec les autorités de ces pays, coordonnaient et réglaient les problèmes pratiques (transports, ...).

Les experts physiciens étaient chargés de former et conseiller les ingénieurs et techniciens affectés à la surveillance radiologique. Ils collaboraient à l'exécution des prélèvements et des mesures et s'assuraient de leur qualité et donc d'une façon générale veillaient au bon déroulement du programme. Ils informaient l'ambassadeur et les responsables français et pouvaient, à la demande de l'ambassadeur et en cas d'absence de l'expert hygiéniste apporter leur aide en cas de situation délicate. Ils assistaient également le responsable de l'ambassade chargé du fonctionnement de la station du réseau mondial.

Les experts hygiénistes avaient un rôle important dans l'interprétation des résultats, en particulier leur interprétation sanitaire. Ils jouaient un rôle de formation et de conseil et intervenaient, en particulier avec l'ambassade, pour éviter toute mauvaise exploitation, volontaire ou non, faite notamment par les médias. Pour cela, ils pouvaient s'appuyer sur des données objectives obtenues par les organismes nationaux compétents.

\subsubsection{La surveillance}

En dehors des filtres qui recueillaient les poussières atmosphériques et des dépôts (précipitations), les prélèvements portaient classiquement sur des produits alimentaires (lait, salades, légumes divers), sur des produits du milieu naturel (par exemple l'herbe de pâturage) ou encore sur des poissons. Les mesures étaient du type de celles qui sont pratiquées dans les laboratoires du DPS (voir en particulier § 3.1.).

Les résultats étaient fournis quotidiennement aux autorités locales et sur un rythme hebdomadaire aux autorités françaises sauf en cas d'évènements particuliers qui justifient une information immédiate. Au terme de sa mission chaque expert rédigeait un rapport d'ensemble.

\subsubsection{La formation d'experts étrangers en France}

En parallèle, des ingénieurs et techniciens des pays concernés étaient accueillis en France pour des stages de formation. Si une majorité d'entre eux ont axé leur formation sur la surveillance radiologique, certains ont souhaité une formation dans d'autres domaines et ont effectué leur stage dans d'autres unités du CEA que le DPS. 


\subsubsection{Les conséquences positives}

Il est indéniable que ce vaste programme a largement contribué à désamorcer des campagnes d'hostilité vis-à-vis de la France. Il faut rappeler qu'en juin 1972, s'est tenue à Guyaquil une conférence réunissant des représentants des cinq pays et d'une délégation française, dont les conclusions ont été très favorables.

Il a permis également de créer de nombreux contacts générant une coopération bilatérale qui s'est poursuivie bien au-delà de l'arrêt du programme. On peut citer notamment :

- en Bolivie, l'aménagement d'une usine pilote de lixiviation de l'uranium et sa mise en conformité avec les exigences de la radioprotection ainsi que l'installation d'un accélérateur générateur de neutrons au centre d'études nucléaires de Viacha (près de La Paz), ou encore un laboratoire de radio agronomie ;

- en Équateur, un irradiateur de $2000 \mathrm{Ci}(74 \mathrm{TBq}) \mathrm{de}{ }^{60} \mathrm{Co}$ a été mis en service en 1981 et est toujours en activité ;

- au Pérou de très nombreuses formations de radioprotection en milieu hospitalier ont été effectuées ;

- au Chili s'est développée une importante coopération pour la mise en service et l'exploitation de leur premier réacteur (La Reina).

\section{Le bilan : exploitation et fonctionnement du dispositif}

\subsection{Conception et fonctionnement du dispositif}

Le dispositif mis en place pour la surveillance de l'environnement associé aux essais nucléaires atmosphériques en Polynésie française a dans son ensemble satisfait aux objectifs qui lui avaient été assignés.

C'est le cas pour sa conception, dans la mesure où il a couvert très largement, et même au-delà, toutes les zones géographiques et les situations où des conséquences radiologiques étaient susceptibles de survenir. L'importance de cette couverture a été adaptée à l'importance et à la probabilité d'occurrence de ces conséquences : en fonction de l'éloignement par rapport aux sites de tir, le réseau de surveillance a été de moins en moins dense de la même façon que sa densité était renforcée en fonction de la densité de la population : c'est le cas de Tahiti, l'île de loin la plus peuplée où la surveillance a été renforcée en dépit de la distance qui la sépare des sites de tirs. D'autres critères ont été pris en compte, comme l'effet barrière de la Cordillère des Andes pour certains pays sud américains. Enfin il faut noter que tous les maillons de l'environnement susceptibles d'intervenir ont été considérés, qu'il s'agisse du milieu continental ou marin, végétal ou animal. 
Il en va de même s'agissant de son fonctionnement. Le principe de base qui voulait que plusieurs acteurs interviennent afin d'éviter toute situation de monopole d'un même organisme, civil ou militaire, ayant eu seul la responsabilité de la mise en place de la surveillance, de la collecte des échantillons, des analyses et mesures et de leur exploitation a été respecté. La qualité des matériels et des personnels a été considérée comme essentielle et des efforts ont toujours été faits pour chercher encore à améliorer cette qualité en fonction de l'expérience acquise et des nécessités nouvelles. Pour garantir la validité des résultats des analyses et mesures, il a été procédé à l'organisation d'inter comparaisons avec des laboratoires reconnus internationalement, aussi bien pour les laboratoires du SMSR que pour ceux du DPS : intercomparaison sous l'égide de l'AIEA pour les premiers, inter comparaisons avec le laboratoire du SCPRI, centre international de référence de l'Organisation mondiale de la santé (OMS) pour les seconds. À ceci il faut ajouter les mesures faites par d'autres organismes, notamment néozélandais, sur des prélèvements qu'ils avaient eux-mêmes effectués.

Enfin, la France a fait preuve, vis-à-vis de ce programme d'une certaine transparence bien qu'il soit compréhensible que l'on touchait là au domaine de la défense et de la sécurité, domaine couvert par le secret dans toutes les nations.

Cette transparence s'est manifestée d'une part par une large diffusion des résultats obtenus, et d'autre part par l'ouverture des laboratoires à des visiteurs, généralement des scientifiques français et étrangers.

\subsection{Utilisation des résultats}

La question de l'utilisation des résultats doit être posée en fonction des objectifs fixés initialement: l'information et la détection rapide et l'évaluation d'un éventuel risque radiologique.

\subsubsection{L'information}

L'information a été un souci permanent et c'est à ce souci que l'on doit les différents rapports régulièrement établis pour rendre compte de la situation radiologique. Ces rapports étaient conçus pour trois destinataires :

- les autorités et les responsables français qui, bien entendu devaient être informés rapidement, complètement et régulièrement. Ces rapports avaient ou pouvaient avoir, une certaine confidentialité puisqu'ils concernaient des zones et des actions sensibles ;

- les responsables polynésiens et les représentants du peuple polynésien qui manifestaient leur inquiétude au regard des expérimentations ; 
- enfin, le milieu scientifique, notamment international, avec l'envoi chaque année à l'UNSCEAR d'un rapport rassemblant tous les résultats, en dehors des sites de tirs, et leur exploitation. Ce rapport annuel, édité d'abord sous le timbre « République française » et le titre « retombées radioactives à la suite des tirs nucléaires en Polynésie française » a été par la suite édité par l'IPSN puis par l'IRSN, sous le titre «surveillance de la radioactivité en Polynésie française et autres pays et territoire » puis «surveillance de la radioactivité en Polynésie française ». L'UNSCEAR a en effet consacré aux essais nucléaires atmosphériques une part importante des rapports qu'il a publié régulièrement à partir de 1958, tout au moins durant la période où ils ont été la source principale, en dehors de l'irradiation naturelle, de l'exposition des populations. Comme il le fait pour l'ensemble des grandes sources d'exposition, l'UNSCEAR procédait à une compilation des données publiées par les différentes nations, dont la France, et en faisait l'exploitation. Toutes ces informations figurent dans les différents rapports UNSCEAR (1977, 1982, 1988, 1993, 2000).

\subsubsection{L'exploitation dosimétrique}

L'autre utilisation des résultats, qui n'est pas la moins importante, loin de là, était la mise en évidence d'éventuelles situations où, en dépit des garanties météorologiques prises, une fraction du nuage suivait une trajectoire qui l'amenait sur une zone habitée, ceci dans le cas du territoire polynésien bien entendu.

Pour de telles situations et d'une façon plus générale pour toutes les populations susceptibles d'être concernées, les résultats de la surveillance pouvaient permettre d'apprécier l'exposition résultante. Concernant la Polynésie française, tous les résultats détaillés et leur exploitation sont présentés par ailleurs (Bataille et Revol, 2002 ; Ministère de la Défense, 2007 ; Martin, 2007).

\subsubsection{La synthèse de l'UNSCEAR}

Les données contenues dans les rapports adressés à l'UNSCEAR ont été, tout au moins pour une partie d'entre elles, intégrées à celles provenant des autres pays. L'exploitation des informations et des résultats qui ont été rassemblés a d'abord permis au comité de procéder à une évaluation, affinée par la suite, du terme source, c'est-à-dire de l'ensemble des radionucléides injectés dans l'atmosphère, puis des grands mouvements qu'ils ont suivis dans leur distribution stratosphérique et troposphérique spécifique à chaque hémisphère et enfin des dépôts au sol. À partir de là, a été évaluée l'exposition externe et, en utilisant des modèles appropriés, les expositions internes pour obtenir les engagements de dose collectifs. 
Les conséquences au niveau régional et local ont été traitées de façon moins complète. Toutefois, en ce qui concerne les essais français en Polynésie des informations sont données quant à la qualité des sites, les précautions prises, ainsi que sur les conséquences des nuages dérivés au niveau de Tureia, des Gambiers et de Tahiti. L'évaluation de la dose collective effective pour la Polynésie inclut les conséquences de tous les essais, français et étrangers. Toutes ces informations sont présentées les rapports de l'UNSCEAR principalement ceux de 1977, 1982, 1988, 1993 et 2000 (UNSCEAR, 1977, 1982, 1988, 1993, 2000).

\section{Conclusion}

La présentation du dispositif mis en place par la France pour exercer une surveillance de l'environnement indispensable à l'évaluation d'éventuelles conséquences radiologiques à partir de données fiables et contrôlables est sans doute encore incomplète dans ses détails, mais elle permet une vision globale des différents problèmes qui se sont posés et des solutions qui ont été apportées. Dans toute la mesure du possible, on a recherché la transparence tant au niveau de la fourniture des informations que de l'accueil des scientifiques, français ou étrangers. La situation présentée est celle qui était rendue nécessaire par des campagnes d'essais dans l'atmosphère. Celles-ci ayant cessé le dispositif a certes évolué, mais d'une part a été adapté aux campagnes d'essais souterrains et d'autre part au suivi des conséquences des essais aériens. Enfin, il faut signaler que les actions entreprises ont permis d'améliorer les connaissances relatives au milieu continental et marin, végétal et animal et ont donné lieu à des études à caractère scientifique très utiles.

\section{RÉFÉRENCES}

Bataille C., Revol H. (2002) Les incidences environnementales et sanitaires des essais nucléaires effectués par la France entre 1960 et 1996 et éléments de comparaison avec les essais des autres puissances nucléaires, rapport de l'Assemblée nationale $n^{\circ} 3571$ et du Sénat n 207.

Martin G. (2007) Les atolls de Mururoa et Fangataufa (Polynésie française). Les expérimentations nucléaires : aspects radiologiques, rapport CEA-R-6136.

Ministère de la Défense (2007) La dimension radiologique des essais nucléaires français en Polynésie À l'épreuve des faits, rapport du ministère de la défense 2007.

UNSCEAR (1977) Sources et effets des rayonnements ionisants, rapport à l'assemblée générale et annexes, Nations-Unies, New-York, 1978.

UNSCEAR (1982) Sources et effets des rayonnements ionisants, rapport à l'assemblée générale et annexes, Nations-Unies, New-York, 1985.

UNSCEAR (1988) Sources et effets des rayonnements ionisants, rapport à l'assemblée générale et annexes, Nations-Unies, New-York, 1988.

UNSCEAR (1993) Sources et effets des rayonnements ionisants, rapport à l'assemblée générale et annexes, Nations-Unies, New-York, 1993.

UNSCEAR (2000) Sources et effets des rayonnements ionisants, rapport à l'assemblée générale et annexes, Volume 1, Nations-Unies, New-York, 2000. 